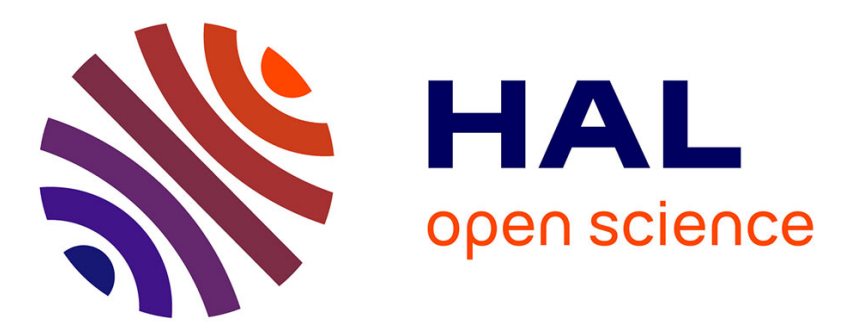

\title{
CSF Amyloid-beta 38 as a novel diagnostic marker for dementia with Lewy bodies
}

Ezra Mulugeta, Elisabet Londos, Clive Ballard, Guido Alves, Henrik Zetterberg, Kaj Blennow, Ragnhild Skogseth, Lennart Minthon, Dag Aarsland

\section{- To cite this version:}

Ezra Mulugeta, Elisabet Londos, Clive Ballard, Guido Alves, Henrik Zetterberg, et al.. CSF Amyloidbeta 38 as a novel diagnostic marker for dementia with Lewy bodies. Journal of Neurology, Neurosurgery and Psychiatry, 2010, 82 (2), pp.160. 10.1136/jnnp.2009.199398 . hal-00589971

\section{HAL Id: hal-00589971 https://hal.science/hal-00589971}

Submitted on 3 May 2011

HAL is a multi-disciplinary open access archive for the deposit and dissemination of scientific research documents, whether they are published or not. The documents may come from teaching and research institutions in France or abroad, or from public or private research centers.
L'archive ouverte pluridisciplinaire HAL, est destinée au dépôt et à la diffusion de documents scientifiques de niveau recherche, publiés ou non, émanant des établissements d'enseignement et de recherche français ou étrangers, des laboratoires publics ou privés. 


\section{CSF Amyloid-beta 38 as a novel diagnostic marker for dementia with Lewy bodies.}

Ezra Mulugeta ${ }^{1,2}$, Elisabet Londos ${ }^{3}$, Clive Ballard ${ }^{2}$, Guido Alves ${ }^{4}$, Henrik Zetterberg ${ }^{5}$, Kaj Blennow $^{5}$, Ragnhild Skogseth ${ }^{6}$, Lennart Minthon $^{3},{ }^{*}$ Dag Aarsland ${ }^{1}$

${ }^{1}$ Department of Old Age Psychiatry, Psychiatric Clinic, Stavanger University Hospital, Norway, ${ }^{2}$ Wolfson Centre for Age Related Diseases, King's College London, UK

${ }^{3}$ Research Unit, Department of Clinical Sciences, Malmö, University of Lund, Sweden

${ }^{4}$ The Norwegian Centre for Movement Disorders, Stavanger University Hospital, Norway

${ }^{5}$ Institute of Neuroscience and Physiology, Department of Psychiatry and Neurochemistry, the Sahlgrenska Academy at the University of Gothenburg, Molndal, Sweden.

${ }^{6}$ Institute of Clinical Medicine, University of Bergen, Norway

Correspondence to:

\section{* Dag Aarsland}

Department of Old Age Psychiatry

Psychiatric Clinic, Stavanger University Hospital

PO Box 8100

N-4068 Stavanger

Norway

E-mail: daarsland@gmail.com Telephone: +47 515150 62, Fax: +47 51515515

Word count: Abstract: 245, Full paper including References 4027

Keywords: Dementia with Lewy bodies, biomarker, cerebrospinal fluid, A $\beta 38$, A $\beta 42, \mathrm{~T}-\mathrm{tau}, \mathrm{P}-$ tau Alzheimer's disease, Parkinson's disease 


\begin{abstract}
Background

The clinical distinction between Alzheimer's disease (AD) and dementia with Lewy bodies (DLB) is sometimes difficult, particularly in mild cases. Although cerebrospinal fluid (CSF) markers such as A 342 and P-tau can distinguish between $\mathrm{AD}$ and normal controls, their ability to distinguish between $\mathrm{AD}$ and DLB is not adequate.
\end{abstract}

\title{
Objective
}

This study aims at investigating whether CSF markers, in particular the level of A $\beta 38$, can differentiate between mild $\mathrm{AD}$ and DLB.

\section{Methods}

In total 85 individuals were included after standardized diagnostic procedures: 30 diagnosed as probable AD, 23 probable DLB, 20 with probable Parkinson's disease dementia (PDD), and 12 non-demented controls subjects. CSF levels of $A \beta 38, A \beta 40$ and $A \beta 42$ were determined using commercially available Ultra-Sensitive multi-array kit assay (MSD) for human A $\beta$ peptides. Total-tau (T-tau) and Phosphorylated tau (P-Tau) were analysed using ELISA (Innotest). In addition, combinations ( $\mathrm{A} \beta 42 / \mathrm{A} \beta 38, \mathrm{~A} \beta 42 / \mathrm{A} \beta 40, \mathrm{~A} \beta 42 / \mathrm{P}$-tau, and $\mathrm{A} \beta 42 / \mathrm{A} \beta 38 / \mathrm{P}-\mathrm{tau})$ were assessed.

\section{Results}

Significant between-group differences were found for all CSF measures, and all except A $\beta 40$, $\mathrm{A} \beta 42$, and $\mathrm{A} \beta 42 / \mathrm{P}$-tau differed between $\mathrm{AD}$ and DLB. $\mathrm{A} \beta 42 / \mathrm{A} \beta 38$ ratio was the measure that best discriminated between $\mathrm{AD}$ and DLB (AUC 0.781; $\mathrm{p}<0.005$ ), with sensitivity $74 \%$ and specificity $77 \%$.

\section{Conclusion}

This study suggests that the level of A $\beta 38$ can potentially contribute in the diagnostic distinction between AD and DLB when combined with A $\beta 42$. Single measures had low diagnostic accuracy, suggesting that developing a panel of markers is the most promising strategy. Studies with independent and larger samples and a priori cut-offs are needed to test this hypothesis. 


\section{Introduction}

Dementia with Lewy Bodies (DLB) is a common dementia with a complex clinical presentation, reduced quality of life and higher costs [1, 2], higher mortality [3], poorer drug response [4], and increased risk for nursing home admission [5] compared to Alzheimer's disease (AD). Identifying people with DLB is therefore crucial, but can be difficult, in particular in early cases where the clinical features are less characteristic.[6] There is therefore a need for biomarkers to aid in the distinction between DLB and AD. However, with the exception of dopamine transporter SPECT, which is expensive and not available at all centres, there are yet no such established biomarkers.[7]

Potential biomarkers should be based on the underlying pathology of the disease. In AD, accumulation of amyloid peptides (A $\beta$-peptides) in plaques as well as species of microtubuleassociated axonal protein tau, which is deposited in tangles, are key pathological features. The A $\beta$-peptides are derived from amyloid precursor protein (APP) by sequential cleavage involving proteolytic enzymes $\beta$ - and $\gamma$ secretaces.[8] The $A \beta 42$ peptide variant is prone to aggregation, is predominantly deposited in senile plaques and is shown to be neuro-toxic.[9]

Hyper phosphorylated tau (P-tau) is one of the major components of neurofibrillary tangles.[10] Lewy bodies, consisting mainly of alpha-synuclein deposits, are the characteristic feature of DLB, although AD type changes are also common.[11]

Cerebrospinal fluid (CSF) A $\beta 42$ together with hyper-phosphorylated tau (P-tau) have been shown to identify incipient AD with good accuracy.[12, 13] However, these peptides discriminate less well between $\mathrm{AD}$ and other dementia subtypes, including DLB.[12, 13] In a study using Western blot and quantitative analyses, Bibl and co-workers showed that by expanding the number of amyloid species by including carboxy-terminally truncated peptides such as $A \beta 37, A \beta 38$, and $A \beta 39$, such markers might provide useful biomarker to distinguish $A D$ from other dementias.[14, 15] In subsequent studies using ELISA, the same group reported that $\mathrm{A} \beta 38$ and the $\mathrm{A} \beta 42 / \mathrm{A} \beta 38$ ratio, have better discriminative power between $\mathrm{AD}$ and other dementias than $A \beta 42$ alone.[15, 16] However, in the latter study, only five patients had DLB. The objective of this paper was therefore to explore whether $\mathrm{A} \beta 38$ and combinations including 
A 338 could distinguish between $\mathrm{AD}$ and DLB, using a larger sample size with DLB, and including normal controls and Parkinson's disease dementia (PDD) as comparison groups.

\section{Methods}

\section{Subjects}

Patients were drawn from two cohorts: The Norwegian DemWest study $(n=42)$ recruited referrals to psychiatry, neurology, and geriatric medicine clinics in Rogaland and Hordaland counties who were diagnosed with mild dementia (i.e Mini-Mental State Examination (MMSE) 220) between 2005-2007.[17] For this study, only patients diagnosed as probable AD (n=30), probable DLB $(\mathbf{n}=9)$ or PDD $(\mathbf{n}=3)$ who consented to lumbar puncture were included. In addition, patients with PDD $(\mathbf{n}=17)$ and probable DLB $(\mathbf{n}=14)$ who were screened between 2006-2008 to participate in a clinical trial of memantine [17] and who consented to lumbar puncture were recruited from the Department of Clinical Sciences, Malmö, University of Lund, Sweden. Subjects without known brain disease who underwent lumbar puncture during orthopaedic surgery or neurologic outpatient assessment with a minimum MMSE score of >24 at the Stavanger University Hospital were recruited as non-demented controls.

\section{Diagnostic procedures}

Diagnostic procedures are described in detail elsewhere.[17, 18] In brief, patients were diagnosed as DLB if they fulfilled clinical consensus criteria for probable DLB [19], probable AD [20] or probable PDD [21] after a detailed clinical assessment by a registered specialist in psychiatry, neurology or geriatric medicine, using standardised assessments for parkinsonism[22] psychiatric symptoms including visual hallucinations (Neuropsychiatric Inventory, NPI)[23], and fluctuating cognition.[24, 25] Blood samples and brain imaging (CT or MRI) were taken from all patients, and a subset of DLB patients underwent dopamine transporter SPECT scans.

\section{Pre-analytical treatment of CSF}

Lumbar puncture (LP) was performed in the L3-L4 or L4-L5 interspace and CSF sampling was performed in all cases between 7-10 am in order to minimize diurnal variation of the level of 
CSF A $\beta$ [26]. The first 3-4mLs of the CSF were dedicated for routine analyses for assessment of relevant CSF abnormalities. Thus, samples were immediately sent on ice to the routine laboratory where routine assay for cell counts, levels of glucose and protein were performed. Study samples were collected in separate polypropylene tubes, and centrifuged at $2000 \mathrm{xg}, 4^{\circ} \mathrm{C}$ for $10 \mathrm{~min}$ to get rid of cell debris and other insoluble materials. Following centrifugation, samples were aliquoted and immediately frozen at $-80^{\circ} \mathrm{C}$ until analyses were performed. Samples from the DemWest study, Stavanger, were originally stored in larger volumes, thus the portions of samples used in this study were aliquots derived from samples frozen and thawed (on ice) once.

\section{Tri-plex human CSF Aß38, Aß40 and Aß42Assay}

All CSF analyses were performed randomized and in duplicates the same day by one of the authors (EM), blinded to clinical information. CSF levels of $A \beta 42, A \beta 40$, and $A \beta 38$ were determined using the $A \beta$ triplex assay (Human $A \beta$ peptide Ultra-Sensitive Kits) developed by Meso Scale Discovery, Gaithersburg, Maryland, USA. This assay uses C-terminus specific antibodies to capture the different $A \beta$ peptides and a SULFO-TAG TM-labeled anti-A $\beta$ antibody (4G8) for detection with electrochemiluminescence.

The tri-plex assay was performed on CSF samples from patients and control subjects as well as standards of specific markers in duplicate and according to the manufacturer's instructions. Briefly, the assay technology is based on MULTI-ARRAY ${ }^{\circledR}$ technology combining electrochemiluminescence detection and patterned arrays offering combination of sensitivity and dynamic range. The triplex assay mentioned here utilizes peptide specific antibodies to capture $A \beta 38, A \beta 40$ and $A \beta 42$ peptides present in CSF. The CSF content of each peptide was then detected by SULFO-TAG-labeled 4G8 detection antibodies. The standard ranges for Aß38 and $A \beta 42$ were 4-3000pg/mL respectively, and for $A \beta 4027.4-20000 \mathrm{pg} / \mathrm{mL}$. The lower limit of detection and limit of quantitation (LLOD/LOQ) for all three analytes were A 338 $(8.69 /<25 \mathrm{pg} / \mathrm{mL}), \mathrm{A} \beta 40(1.28 / \sim 50 \mathrm{pg} / \mathrm{ml})$ and $\mathrm{A} \beta 42(12.37 / \sim 35 \mathrm{pg} / \mathrm{mL}) \mathrm{pg} / \mathrm{mL}$ respectively. To determine inter and intra assay variations we included different CSF samples (1= low level, $2=$ medium level) in replicates as run controls. Within-assay precision for replicated samples on same plate (intra-assay) variation for the "low level" sample and for individual 
analytes; $A \beta 38, A \beta 40$ and $A \beta 42$ were between 5-6\%. The inter-assay variability, (same sample analysed on different plates) for the "low level" sample and each of the analytes $A \beta 38, A \beta 40$ and $A \beta 42$ was 9,13 and $8 \%$ respectively. Similarly the intra-assay variability for the "medium level sample" and each of the analytes mentioned above were between 4$7 \%$ and the inter-assay variability for "medium level sample" and each of the analytes were 15,14 and $12 \%$ respectively.

\section{ELISA of total and phosphorylated tau}

CSF T-tau \& P-tau

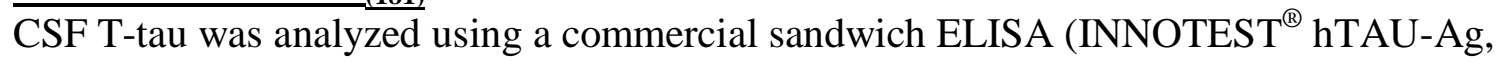
Innogenetics, Gent, Belgium) specifically constructed to measure all tau isoforms irrespective of phosphorylation status, as previously described [27]. In this assay, the monoclonal antibody AT120 was used for capture, while the biotinylated monoclonal antibodies HT7 and BT2 were used as detection antibodies. AT120 and HT7 both react equally well with both normal and hyperphosphorylated, while BT2 preferentially recognizes normal tau protein. The standard range of the T-tau assay was 75-1200 pg/ml, the LOD was $59 \mathrm{pg} / \mathrm{ml}$, and intraand inter-assay CVs ranged from 1.2-5.9\% and 1.7-6.0\% respectively.

CSF P-tau was measured using a commercial sandwich ELISA method (INNOTEST ${ }^{\circledR}$ PHOSPHO-TAU $_{(181 \mathrm{P})}$, Innogenetics, Ghent, Belgium), as described previously [28]. In this assay, the monoclonal antibody HT7 was used for capture and the biotinylated monoclonal antibody AT720 as detector antibody. HT7 both react equally well with both normal and hyperphosphorylated, while AT270 specifically reacts with tau phosphorylated at threonine-181. The P-tau assay had a standard range of $15.6-500$ pg/ml, a LOD of 15.6 $\mathrm{pg} / \mathrm{ml}$, and intra- and inter-assay CVs of $<5 \%$ and $<10 \%$ respectively.

\section{Statistics}

Values of CSF markers were expressed as absolute (pg/ml). In addition to the single markers, pre-specified combinations were analysed: $A \beta 42 / A \beta 40, A \beta 42 / \mathrm{P}-\operatorname{tau}, A \beta 42 / A \beta 38$, and $A \beta 42 / A \beta$ 38/P-tau. Since measures were not normally distributed, median and interquartile range (IQR) were expressed, and Spearman correlations were performed. Between-group comparisons were 
made using Kruskall-Wallis and chi square tests. Pre-planned post-hoc pair-wise comparisons (Mann-Whitney) between AD and DLB were performed subsequently. The global diagnostic accuracies were assessed by the received-operated characteristic curve (AUC). Since this was a hypothesis-generating study, a $p$ value of $<0.05$ was considered significant, and no attempts to adjust for multiple comparisons were made. Cut-off points and sensitivity and specificity were determined based on the coordinate points of the curves. Positive (sensitivity/1-specificity) and negative (specificity/1-sensitivity) likelihood ratios (LR) were calculated. Cut-off points and sensitivity and specificity were determined based on the coordinate points of the curves.

\section{Results}

\section{Characteristics}

In total, 85 subjects participated: AD (n=30), DLB $(n=23)$, PDD $(n=20)$ and normal controls $(n=12)$. The characteristics of the subjects are shown in table 1 . The groups did not differ in terms of age, and the dementia groups did not differ regarding MMSE score. As expected, there were more males in the DLB and PDD groups compared to the other groups, and the AD and DLB groups differed significantly (chi square 4.6, $\mathrm{p}=.03$ ). The disease duration differed significantly, with a longer duration in DLB than AD $(p=.042)$.

Table 1. Characteristics of the groups

\begin{tabular}{|l|l|l|l|l|l|}
\hline & NC & AD & DLB & PDD & P \\
\hline $\mathrm{N}$ & 12 & 30 & 23 & 20 & \\
\hline Age & $73.5(16.8)$ & $75.5(11.3)$ & $74(10.8)$ & $73(11)$ & .89 \\
\hline MMSE & $28.5(1.8)$ & $23.5(4.3)$ & $23(7.8)$ & $23(9)$ & $<.0005$ \\
\hline Gender, M/F* & $4 / 8$ & $12 / 18$ & $16 / 7$ & $13 / 7$ & $=.055$ \\
\hline UPDRS motor & ND & $0(2)$ & $30.5(32.5)$ & $34.5(17.3)$ & $<.0005$ \\
\hline $\begin{array}{l}\text { Duration of } \\
\text { disease }\end{array}$ & NA & $2(2)$ & $3.5(3)$ & $8(4)$ & $<.0005$ \\
\hline
\end{tabular}

Numbers represent median and inter-quartile range or *number of people and \%

P values based on Kruskall-wallis test; ND: Not done NA: not applicable

$\mathrm{NC}=$ Normal control, $\mathrm{AD}=$ Alzheimer's disease, $\mathrm{DLB}=$ Dementia with Lewy bodies, PDD=Parkinson's disease dementia 
CSF measures and associations with diagnosis and other characteristics

The CSF values are shown in table 2 and Figure 1. There were significant between-group differences on all markers. Compared to NC; the pattern in $\mathrm{AD}$ was as expected, with low A $\beta 42$ and high T-tau and P-tau. Significant differences between DLB and AD were observed for all measures except $A \beta 40$ and $A \beta 42$, and $A \beta 42 / P$-tau. Whereas the single markers were changed in the same direction in $\mathrm{AD}$ and DLB, a different picture emerged for the combined markers involving A $\beta 38$, which were decreased in AD but increased in DLB and PDD compared to NC. Mean CSF concentrations did not differ between the Norwegian and Swedish DLB/PDD patients. The AUC analyses demonstrated that the $A \beta 42 / A \beta 38$ ratio was the strongest marker for differentiation between $\mathrm{AD}$ and DLB, with an AUC of 0.732 (95\% CI 0.587-0.876) (p=.007), with sensitivity $74 \%$ and specificity $67 \%$ at cut-off 0.50 . Positive LR was 2.2 (95\% CI $1.3-3.6$ and negative LR $0.3(0.25-0.8)$.

There was a trend towards association between $A \beta 38$ and gender $(p=0.061)$. Since there were gender differences between $\mathrm{AD}$ and DLB, the analyses were therefore performed for each gender separately. The overall analyses were confirmed in males, whereas no significant differences were found in the smaller female group (data not shown). When all subjects were included, age correlated with total tau (rho 0.29, p=.007), $\mathrm{A} \beta 38$ (0.24, p=.029), and $\mathrm{A} \beta 40(0.26, \mathrm{p}=.015)$, and all three A $\beta$ species correlated significantly with MMSE score (rho 0.24-0.31, p $0.004-0.03$ ).

Finally, $\mathrm{A} \beta 38$ correlated with duration of disease $(0.25, \mathrm{p}=.04)$. Including patients only, significant correlations with age were found for total tau ( rho $=0.25, p<.05$ ), A $\mathrm{A42}$ correlated with UPDRS motor score $(0.37, p=.04)$, and duration with A $\beta 38$ (rho=-0.26, p=.032), whereas a non-significant trend between MMSE and A $\beta 38$ was found (rho=0.22,

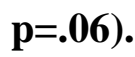


Table 2. CSF concentration of protein markers in the diagnostic groups

\begin{tabular}{|l|l|l|l|l|l|l|l|}
\hline Markers & NC & AD & DLB & PDD & P1 & P2 & AUC \\
\hline A $\beta 38(\mathrm{pg} / \mathrm{mL})$ & $635(712)$ & $440(305)$ & $385(244)$ & $404(299)$ & .005 & .013 & 0.70 \\
\hline $\mathrm{A} \beta 40(\mathrm{pg} / \mathrm{mL})$ & $8286(6652)$ & $5461(2632)$ & $5507(2109)$ & $5036(3633)$ & .048 & .36 & 0.43 \\
\hline $\mathrm{A} \beta 42(\mathrm{pg} / \mathrm{mL})$ & $337(378)$ & $192(74)$ & $223(163)$ & $287(107)$ & .001 & .84 & 0.52 \\
\hline T-tau $(\mathrm{pg} / \mathrm{mL})$ & $250(166)$ & $382(363)$ & $303(116)$ & $303(122)$ & .023 & .009 & 0.68 \\
\hline P-tau $(\mathrm{pg} / \mathrm{mL})$ & $58.3(35.4)$ & $86.5(61)$ & $60.2(52.8)$ & $57.1(41.3)$ & .027 & .011 & 0.69 \\
\hline $\mathrm{A} \beta 42 / \mathrm{A} \beta 38$ & $0.62(0.14)$ & $0.46(0.29)$ & $0.66(0.34)$ & $0.74(0.28)$ & .0005 & .001 & 0.77 \\
\hline $\mathrm{A} \beta 42 / \mathrm{A} \beta 40$ & $0.058(0.02)$ & $0.032(0.02)$ & $0.043(0.02)$ & $0.055(0.1)$ & .002 & .004 & 0.64 \\
\hline $\mathrm{A} \beta 42 \times 1000 / \mathrm{A} \beta 38 / \mathrm{P}-\mathrm{tau}$ & $9.2(6.3)$ & $4.8(9.5)$ & $10.8(11.1)$ & $11.4(15.2)$ & .0005 & .015 & 0.74 \\
\hline $\mathrm{A} \beta 42 / \mathrm{P}-\mathrm{tau}$ & $9.0(2.9)$ & $2.1(2.0)$ & $3.9(5.0)$ & $4.8(2.7)$ & .0005 & .076 & 0.71 \\
\hline
\end{tabular}

$\mathrm{NC}=$ non-demented control subjects, $\mathrm{AD}=$ Alzheimer's disease, $\mathrm{DLB}=$ Dementia with Lewy

Bodies and PDD = Parkinson's Disease Dementia

Numbers represent median and interquartile rnage $(\mathrm{pg} / \mathrm{ml})$ or ratio

P1=All groups, Kruskall-wallis test; $\mathrm{p} 2=\mathrm{AD}$ vs DLB, Mann-Whitney test

AUC: Area under the curve.

\section{Discussion}

We investigated whether CSF markers could distinguish between mild DLB and AD. The main finding was that the ratio between $\mathrm{A} \beta 42$ and $\mathrm{A} \beta 38$ was the CSF marker that best distinguished between $\mathrm{AD}$ and DLB. However, the accuracy is still well below the $85 \%$ level which is recommended by Consensus Report of the Working Group on: "Molecular and Biochemical Markers of Alzheimer's Disease" for a diagnostic test.[29] Given the increasingly recognised need for an early dementia diagnosis [30], and the difficulties in making an accurate clinical diagnosis of DLB, in particular early in course, these findings are nevertheless encouraging. Our findings further suggest that the pattern from a panel of CSF markers is the best strategy forward, although continued research is needed to develop an adequate biomarker for the differentiation between $\mathrm{AD}$ and DLB. 
There were associations between $A \beta$-peptide species with cognitive impairment, and $A \beta 38$ correlated with disease duration. This is consistent with a recent study demonstrating that the CSF level of alpha-synuclein was associated with dementia severity in DLB,[31] and suggests that CSF markers can be useful markers of the progression of disease pathology, and thus might serve as biological outcome measures in clinical trials of disease modifying therapies.

This study has limitations which need to be considered when interpreting the findings. First, the diagnoses were based on clinical assessment and may thus not be $100 \%$ accurate. However, the two research groups have long experience in diagnosing DLB, and standardised and recommended diagnostic procedures and consensus criteria were used. These have shown good clinico-pathologic correlation [32], suggesting that the diagnostic accuracy is relatively high. Of note, there is also pathological overlap between $\mathrm{AD}$ and $\mathrm{DLB}$, and the pathological classification of the two diseases is still under discussion.[33]

There were gender differences in the expected direction. The overall findings were confirmed in the male patients, supporting the validity of the overall findings. The lack of significant differences in the female patients should be interpreted in light of the low statistical power due to the low number of female DLB patients. Difference in duration of disease might also have introduced a bias. Lumbar puncture was not standardised in relation to meals at one study site (Malmø). This might have influenced the findings [26], although such an effect is most likely to be minor as no differences in mean values were observed in the DLB/PDD group between study centres.

Finally, as most previous studies, we derived cut-offs from the population under study. This procedure has an inherent risk for overestimating diagnostic accuracy.[34] Further studies are therefore need to test the accuracy of a priori determined cut-offs rather than establishing a cutoff directly on the cohort under investigation.

The observation of a similar pattern of CSF markers in DLB and PDD, with largely shared underlying brain changes, adds biological validity to the observations. However, we found somewhat lower diagnostic accuracies for the $\mathrm{A} \beta$ species compared with a previous study.[16]. Furthermore, our findings of Tau and P-Tau, although significantly different between the groups, 
show lower diagnostic accuracy compared to a recent study [35]. Possible explanations for these differences include that we specifically explored the differentiation between AD and DLB, whereas only $10 \%$ of the non-AD group in the previous study were DLB or PDD. Thus, since AD-type brain changes are typically found in DLB [36], this differentiation may be more challenging than distinguishing $\mathrm{AD}$ from frontotemporal and vascular dementias. Secondly, our sample included older patients (age 73-75) with mild dementia, with a mean MMSE score of 2324, compared to younger patients (mean age below 70) with a lower mean MMSE score (below 20) in [16]. Whereas the previous study (35) was based on an autopsy-cohort, we used clinically diagnosed cases with presumed lower diagnostic accuracy. In addition, there were differences in the pre-analytic handling and assay methods between the different studies, possibly leading to variation in CSF marker concentrations between different centres. This was shown in a recent multi-centre study [12], in which some of the Stavanger cases participated. Finally, although there are strong correlations between the different methods and commercially available kits used to determine level of CSF markers, there is considerable variability in the average values of results.

In conclusion, our findings suggest that $\mathrm{A} \beta 38$ should be included in future studies to identify CSF biomarkers to differentiate between AD and DLB. Future studies should also explore whether CSF markers can increase diagnostic accuracy over and above standard clinical assessment, and whether they can predict disease progression. Finally, whether establishing CSF panels combining specific $A \beta$-peptides and tau markers with alpha-synuclein species might improve this differentiation should also be investigated. 


\section{Acknowledgements}

We would like to thank all patients in Western Norway and Malmö, Sweden and control subjects for their willingness to participate in the study. We also thank all personnel (in the different clinics in Western Norway and Malmö, Sweden) for their effort to contribute to this study as well as for collecting clinical data and CSF. We are especially grateful to Hilde Rydland Marianayagam, and Ingrid Langeland Braut for their assistance in collection of CSF from control patients. Special thanks to Sara Hulberg, Sahlgrenska Academy for help with preparation of CSF samples and analyses. Karen Simonsen for excellent administrative support. This study was funded by the Western Norway Regional Health Authority, HelseVest (grant\# 911390).

\section{Licence for Publication}

The Corresponding Author has the right to grant on behalf of all authors and does grant on behalf of all authors, an exclusive licence (or non exclusive for government employees) on a worldwide basis to the BMJ Publishing Group Ltd to permit this article (if accepted) to be published in JNNP and any other BMJPGL products and sublicences such use and exploit all subsidiary rights, as set out in our licence. (http://group.bmj.com/products/journals/instructionsfor-authors/licence-forms)

\section{Competing Interests}

None declared. 


\section{REFERENCES}

1. Bostrom F, Jonsson L, Minthon L, et al. Patients with dementia with lewy bodies have more impaired quality of life than patients with Alzheimer disease. Alzheimer Dis Assoc Disord. 2007;21:150-4.

2. Bostrom F, Jonsson L, Minthon L, et al. Patients with Lewy body dementia use more resources than those with Alzheimer's disease. Int J Geriatr Psychiatry. 2007;22:713-9.

3. Williams MM, Xiong C, Morris JC, et al. Survival and mortality differences between dementia with Lewy bodies vs Alzheimer disease. Neurology. 2006;67:1935-41.

4. Aarsland D, Perry R, Larsen JP, et al. Neuroleptic sensitivity in Parkinson's disease and parkinsonian dementias. J Clin Psychiatry. 2005;66:633-7.

5. Rongve A, Skogseth R, Aarsland D. Risk of nursing home placement in Dementia with Lewy Bodies. ICAD 2009; 2009; Vienna, Austria: The Journal of The Alzheimer's Association; 2009.

6. Tiraboschi P, Salmon DP, Hansen LA, et al. What best differentiates Lewy body from Alzheimer's disease in early-stage dementia? Brain. 2006;129:729-35.

7. Aarsland D, Kurz M, Beyer M, et al. Early discriminatory diagnosis of dementia with Lewy bodies. The emerging role of CSF and imaging biomarkers. Dement Geriatr Cogn Disord. 2008;25:195-205.

8. Mattson MP. Pathways towards and away from Alzheimer's disease. Nature. 2004;430:631-9.

9. Iwatsubo T, Odaka A, Suzuki N, et al. Visualization of A beta 42(43) and A beta 40 in senile plaques with end-specific A beta monoclonals: evidence that an initially deposited species is A beta 42(43). Neuron. 1994;13:45-53.

10. Blennow K, Vanmechelen E, Hampel H. CSF total tau, Abeta42 and phosphorylated tau protein as biomarkers for Alzheimer's disease. Mol Neurobiol. 2001;24:87-97.

11. Lippa CF, Duda JE, Grossman M, et al. DLB and PDD boundary issues: diagnosis, treatment, molecular pathology, and biomarkers. Neurology. 2007;68:812-9.

12. Mattsson N, Zetterberg H, Hansson O, et al. CSF biomarkers and incipient Alzheimer disease in patients with mild cognitive impairment. Jama. 2009;302:385-93.

13. Blennow K. CSF biomarkers for mild cognitive impairment. J Intern Med. 2004;256:22434. 
14. Bibl M, Mollenhauer B, Esselmann H, et al. CSF amyloid-beta-peptides in Alzheimer's disease, dementia with Lewy bodies and Parkinson's disease dementia. Brain. 2006;129:1177-87. 15. Bibl M, Mollenhauer B, Lewczuk P, et al. Validation of amyloid-beta peptides in CSF diagnosis of neurodegenerative dementias. Mol Psychiatry. 2007;12:671-80.

16. Welge V, Fiege O, Lewczuk P, et al. Combined CSF tau, p-tau181 and amyloid-beta 38/40/42 for diagnosing Alzheimer's disease. J Neural Transm. 2009;116:203-12.

17. Aarsland D, Londos E, Ballard C. Parkinson's disease dementia and dementia with Lewy bodies: different aspects of one entity. Int Psychogeriatr. 2009;21:216-9.

18. Aarsland D, Rongve A, Nore SP, et al. Frequency and case identification of dementia with Lewy bodies using the revised consensus criteria. Dement Geriatr Cogn Disord. 2008;26:445-52.

19. McKeith IG, Dickson DW, Lowe J, et al. Diagnosis and management of dementia with Lewy bodies: third report of the DLB Consortium. Neurology. 2005;65:1863-72.

20. McKhann G, Drachman D, Folstein M, et al. Clinical diagnosis of Alzheimer's disease: report of the NINCDS-ADRDA Work Group under the auspices of Department of Health and Human Services Task Force on Alzheimer's Disease. Neurology. 1984;34:939-44.

21. Emre M. Dementia associated with Parkinson's disease. Lancet Neurol. 2003;2:229-37.

22. Fahn S ER, \& Committee., M. o. t. U. D. Unified Parkinson's Disease Rating Scale. NJ: Florham Park, NJ: MacMillan Health Care Information; 1987.

23. Cummings JL, Mega M, Gray K, et al. The Neuropsychiatric Inventory: comprehensive assessment of psychopathology in dementia. Neurology. 1994;44:2308-14.

24. Ferman TJ, Smith GE, Boeve BF, et al. DLB fluctuations: specific features that reliably differentiate DLB from AD and normal aging. Neurology. 2004;62:181-7.

25. Walker MP, Ayre GA, Cummings JL, et al. Quantifying fluctuation in dementia with Lewy bodies, Alzheimer's disease, and vascular dementia. Neurology. 2000;54:1616-25.

26. Bateman RJ, Wen G, Morris JC, et al. Fluctuations of CSF amyloid-beta levels: implications for a diagnostic and therapeutic biomarker. Neurology. 2007;68:666-9.

27. Blennow K, Wallin A, Agren H, et al. Tau protein in cerebrospinal fluid: a biochemical marker for axonal degeneration in Alzheimer disease? Mol Chem Neuropathol. 1995;26:231-45. 
28. Vanderstichele H, De Vreese K, Blennow K, et al. Analytical performance and clinical utility of the INNOTEST PHOSPHO-TAU181P assay for discrimination between Alzheimer's disease and dementia with Lewy bodies. Clin Chem Lab Med. 2006;44:1472-80.

29. Consensus report of the Working Group on: "Molecular and Biochemical Markers of Alzheimer's Disease". The Ronald and Nancy Reagan Research Institute of the Alzheimer's Association and the National Institute on Aging Working Group. Neurobiol Aging. 1998;19:10916.

30. Dubois B, Feldman HH, Jacova C, et al. Research criteria for the diagnosis of Alzheimer's disease: revising the NINCDS-ADRDA criteria. Lancet Neurol. 2007;6:734-46.

31. Ballard C, Jones EL, Londos E, et al. alpha-synuclein antibodies recognize a protein present at lower levels in the CSF of patients with dementia with Lewy bodies. Int Psychogeriatr. 2009:1-7.

32. Fujishiro H, Ferman TJ, Boeve BF, et al. Validation of the neuropathologic criteria of the third consortium for dementia with Lewy bodies for prospectively diagnosed cases. J Neuropathol Exp Neurol. 2008;67:649-56.

33. Dickson DW, Fujishiro H. In dementia with Lewy bodies, Braak stage determines phenotype, not Lewy body distribution. Neurology. 2008;70:2087-8; author reply 8-9.

34. Bossuyt PM, Reitsma JB, Bruns DE, et al. Towards complete and accurate reporting of studies of diagnostic accuracy: the STARD initiative. Bmj. 2003;326:41-4.

35. Koopman K, Le Bastard N, Martin JJ, et al. Improved discrimination of autopsyconfirmed Alzheimer's disease (AD) from non-AD dementias using CSF P-tau(181P). Neurochem Int. 2009;55:214-8.

36. Mori H. Pathological substrate of dementia in Parkinson's disease--its relation to DLB and DLBD. Parkinsonism Relat Disord. 2005;11 Suppl 1:S41-5. 
Figure 1. Diagnostic accuracy for DLB versus $A D$ for $A \beta 42 / A \beta 38$ ratio and $A \beta 42$ as measured using the area under the curve

\section{ROC Curve}

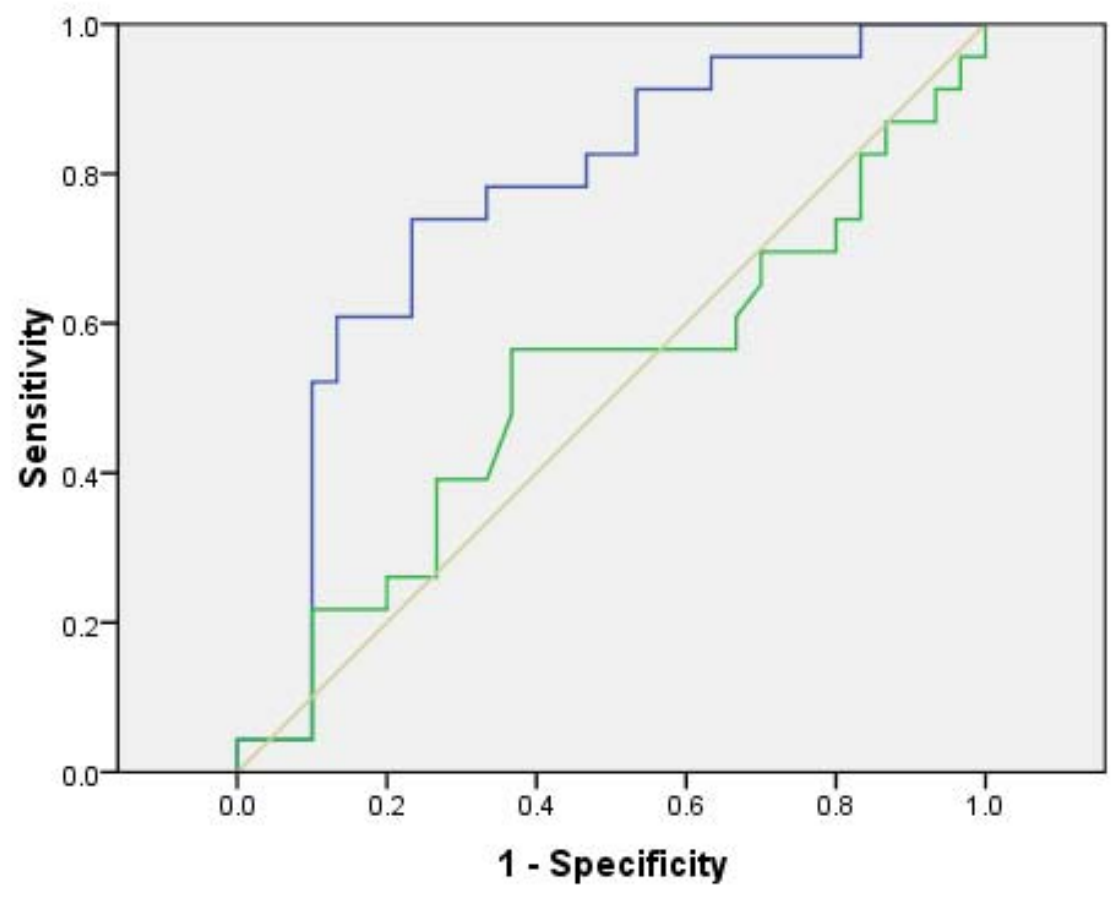

Source of the Curve

- ab42ab38ratio -Ab42_MSD_pgmL Reference Line

Diagonal segments are produced by ties.

Note. $A \beta 42$ A $\beta 38$ ratio is the ratio between CSF $A \beta 42$ and $A \beta 38$.

A $\beta 42 \_$MSD_pgml is the CSF level of A $\beta 42$ in $\mathrm{pg} / \mathrm{ml}$. 